\title{
Safety profile of a single pegylated asparaginase (PEG-ASP) dose in remission induction for acute lymphoblastic leukemia (ALL)
}

\author{
G. HELBIG*, A. ARMATYS, K. BORAL, A.J. KOPINSKA, K. WOZNICZKA, M. DWORACZEK, K. CHROMIK, A. KOCLEGA, M. PANZ-KLAPUCH, \\ M. WYSOCKA, A. JANIKOWSKA
}

Department of Hematology and Bone Marrow Transplantation, School of Medicine in Katowice, Medical University of Silesia, Katowice, Poland

*Correspondence: ghelbig@o2.pl

Received February 14, 2018 / Accepted May 2, 2018

\begin{abstract}
The incorporation of pegylated asparaginase (PEG-ASP) in pediatric and adult acute lymphoblastic leukemia (ALL) protocols remains a worldwide therapeutic approach. However the safety profile remains a challenge, and herein we report the toxicity of an intravenous single dose of $1000 \mathrm{IU} / \mathrm{m} 2$ PEG-ASP administered in remission induction for adult ALL patients. Thirty-two patients at median diagnostic age of 32 years (median of 19-65) were included in this analysis. Most patients had B-cell lymphoblastic leukemia $(n=26 ; 78 \%)$ and $81 \%$ of cases were $<55$ years at study entry. $75 \%$ of patients had $<30 x 109 / 1$ leukocyte count at diagnosis and median follow-up was 14 months (range 0.8-69). All grade 3/4 adverse events (AEs) after PEG-ASP administration were observed in 24 patients (75\%). The most common grade 3/4 AEs were: decreased fibrinogen (58\%), increased bilirubin (31\%) and increased GGTP (27\%). Clinical manifestations related to PEG-ASP were seen in 9 patients and included: abdominal pain $(n=6)$, thrombosis $(n=2)$, diarrhea $(n=1)$ and pancreatitis $(n=1)$. The median time from PEG-ASP administration to first toxic symptoms was 7 days (range 1-19), and there were also 4 (13\%) early induction deaths. All deaths were observed in $\geq 50$-year-old patients after a median of 5 days following PEG-ASP (range 1-9). Three of these four patients had massive obesity. While all expired patients had grade 4 neutropenia and thrombocytopenia at the time of death, sepsis was not present. Administration of PEG-ASP in induction remission for ALL patients resulted in a significant, but mostly reversible hepatotoxicity. This PEG-ASP treatment should be administered with caution for older, obese patients.
\end{abstract}

Key words: acute lymphoblastic leukemia, asparaginase, pegylated asparaginase, toxicity, induction, remission

L-Asparaginase remains a key component of treatment protocols in adult and pediatric ALL patients $[1,2]$. Native (unmodified) asparaginase is extracted from Escherichia coli. The major limitation of its use relates to allergic reactions resulting from antibody production. Pegylated Escherichia coli asparaginase (PEG-ASP) has a longer half-life and is potentially less immunogenic than the native preparation. Moreover, the administration of PEG-ASP causing a prolonged depletion of plasma asparagine has demonstrated anti-leukemic efficacy similar to that of Escherichia coli asparaginase $[1,3]$. The toxicity of PEG-ASP seems to be identical to that in L-ASP except for hypersensitivity reactions which are reported 2-3 times more common in the latter [1]. The toxicity data, including grade $3 / 4$ side effects of PEG-ASP administration in older adults with ALL, are conflicting $[4,5]$.

Herein, we report the safety profile of intravenous single dose of PEG-ASP administered during remission induction in adult ALL patients.

\section{Patients and methods}

Induction treatment. All eligible patients were between 18 and 65 years old and had newly diagnosed B- or T-cell acute lymphoblastic leukemia (ALL). They were recruited between 2011 and 2016 and gave written informed consent before the study. All procedures performed in studies involving human participants were in accordance with the ethical standards of the institutional and/or national research committee, and with the 1964 Helsinki declaration and its later amendments or comparable ethical standards. Patients received treatment according to protocols adopted by the Polish Adult Leukemia Group: ALL6. The treatment stratification was based on patient's age at diagnosis ( $<55$ years and $\geq 55$ years) and the presence of Philadelphia ( $\mathrm{Ph})$ chromosome and/or BCR-ABL transcript. According to ALL6 protocol, the Pegylated Escherichia-coli-derived L-asparaginase (PEG-ASP) was administered only to patients with 
undetectable Ph chromosome and/or BCR-ABL. There was no difference in induction protocols between patients with B- and T-cell ALL.

The treatment protocol. For $\mathrm{Ph}(-)$ patients $<55$ years treatment was; 5-day pre-treatment with prednisone $60 \mathrm{mg} / \mathrm{m}^{2}$ (40 mg/m² for $\geq 40$ and $<55$ years), then continued on days (D) 1-28 followed by induction with vincristine $1.4 \mathrm{mg} / \mathrm{m}^{2}$ and daunorubicin $50 \mathrm{mg} / \mathrm{m}^{2}\left(\geq 40\right.$ years, $\left.40 \mathrm{mg} / \mathrm{m}^{2}\right)$ on D1, D8, D15 and D22. PEG-ASP at $1000 \mathrm{IU} / \mathrm{m}^{2}$ was given on D13. Intrathecal liposomal cytarabine (Depocyte, Mundipharma) at 50mg was administered twice during induction: in the pre-treatment phase and on D10.

For ALL patients $\geq 55$ years, the pre-treatment included dexamethasone $10 \mathrm{mg} / \mathrm{m}^{2}$ for 5 days continued on D1D7 and D15-D21 followed by induction with vincristine $1.4 \mathrm{mg} / \mathrm{m}^{2}$ and daunorubicin $30 \mathrm{mg} / \mathrm{m}^{2}$ on D1, D8, D15 and D22. PEG-ASP at $1000 \mathrm{IU} / \mathrm{m}^{2}$ was administered on D10. Intrathecal Depocyte at $50 \mathrm{mg}$ was given in the pre-treatment phase and on D10.

All patients received antibacterial and antifungal prophylaxis throughout the induction. Granulocyte colony-stimulating factor (G-CSF) was recommended for all neutropenic patients. Antithrombotic prophylaxis was not routinely used and fresh frozen plasma (FFP) was recommended for patients with abnormal clotting tests or bleeding; antithrombin replacement was inaccessible in our center.

Table 1. Patients characteristics at study entry.

\begin{tabular}{lc}
\hline Characteristic & $\mathbf{N}=32$ \\
\hline Age; years, (median; range) & $32(19-65)$ \\
$<55$ years (\%) & $26(81)$ \\
$\geq 55$ years (\%) & $6(19)$ \\
Sex; male/female & $17 / 15$ \\
Lineage B-cell/T-cell & $26 / 6$ \\
Wbc (×10\%/l); (median; range) & $9.1(0.98-392.8)$ \\
$<30(\%)$ & $24(75)$ \\
$30-99.9(\%)$ & $5(16)$ \\
$>100$ (\%) & $3(9)$ \\
Hgb (g/dl); (median; range) & $9.5(5.6-14.3)$ \\
Plt (×10\%/l); (median; range) & $52(3.0-279)$ \\
Blasts cells in PB (\%); (median; range) & $45(0-100)$ \\
Blasts cells in BM (\%); (median; range) & $87(20-100)$ \\
Cytogenetics (\%) & \\
t(4;11) & $3(9)$ \\
$\quad$ complex karyotype & $2(6)$ \\
normal karyotype & $7(22)$ \\
failed & $20(63)$ \\
History of alcohol abuse (\%) & $1(3)$ \\
History of allergy (\%) & $3(9)$ \\
\hline
\end{tabular}

Legend: $\mathrm{Hgb}=$ hemoglobin; Plt=platelets; $\mathrm{Wbc}=$ white blood cells
Toxicity assessment. The Common Terminology Criteria for Adverse Events (CTCAE) version 4.0 assessed toxicity. The Summary of Product Characteristics was used for laboratory and clinical events related to PEG-ASP administration. PEG-ASP antibody and serum asparaginase activity were not measured.

Statistics. The probability of overall survival was calculated by Kaplan-Meier estimate. Induction-related death was defined as any death during induction not primarily related to progressive ALL. All computations were performed with StatSoft Poland analysis software (version 10.0).

\section{Results}

Patient characteristics. Thirty-two patients at median age of 32-year at diagnosis (range 19-65) were included in this retrospective analysis. Most patients had B-cell lymphoblastic leukemia $(n=26 ; 78 \%)$ and $81 \%$ of cases were $<55$ years at study entry. Cytogenetic results were obtained in 12 patients (37\%); and normal diploid karyotype was the most common finding (22\%). Most patients $(n=19 ; 63 \%)$ failed karyotyping and $75 \%$ of patients had leukocyte count $<30 \times 10^{9} / 1$ at diagnosis. The median follow-up was 14 months (range 0.8-69). Antithrombin (AT) concentration was normal in 17 studied patients (median 106\%; range: 79-141). The median prednisone dose or equivalent administered before PEG-ASP was 1750mg (range 210-2600), and median total dose of PEG-ASP in induction was 1800 IU (range 1400-2200). Patient study characteristics are listed in Table 1.

Laboratory and clinical findings after PEG-ASP. All adverse event grades (AE) were present in studied patients, but the following clinical manifestations likely related to PEG-ASP were seen in 9 patients: abdominal pain $(n=6)$, thrombosis $(n=2)$, diarrhea $(n=1)$ and pancreatitis $(n=1)$. Median time from PEG-ASP dose to first symptoms was 7 days (range 1-19).

The following grade 3/4 AEs were observed in 24 patients after PEG-ASP administration (75\%): fibrinogen decrease (58\%), bilirubin increase (31\%) and GGTP increase (27\%). AT was measured in 12 patients after PEG-ASP and it was reduced in 8 (median 54\%; range 28-118). The blood investigation details are in Table 2.

There were $4(13 \%)$ early induction deaths among those receiving PEG-ASP. All deaths were observed in $\geq 50$-year-old patients at diagnosis after median of 5 days after PEG-ASP (range 1-9). Three out of 4 patients had massive obesity (BMI $>40)$ and all expired patients had grade 4 neutropenia and thrombocytopenia at the time of death. They also had slightly elevated CRP levels (median $10.5 \mathrm{~g} / \mathrm{l}$; range: 6.16-29.3) but bacterial/fungal cultures remained negative. (Table 3).

Patients surviving PEG-ASP had at least one grade 3/4 AEs. Induction chemotherapy was discontinued in 4 patients and the subsequent dose of daunorubicin and vincristine was delayed in the remaining 3 cases. Nine patients received 
anticoagulants (low molecular weight heparin) for thrombosis $(\mathrm{n}=2)$ or as prophylaxis $(\mathrm{n}=7)$, and the median transfused fresh frozen plasma units was 8 (range 3-17).

Complete remission rate and survival. The complete remission (CR) rate for the entire cohort after induction was $81 \%(n=26)$, and there were the 4 patients with early deaths and 2 cases with incomplete induction treatment due to complications. One of those latter achieved CR after a second induction and the other remained primary resistant.

At the last follow-up, 18 of the 26 patients with first induction CR are still alive (69\%). All those patients underwent autologous or allogeneic stem cell transplantation depending on risk factors. The remaining 8 patients died of disease relapse $(n=6)$ or due to infectious complications after allogeneic stem cell transplantation $(n=2)$. The median survival is 37 months and the 2-year probability of overall survival is $78 \%$. The flow chart showing response rates and disease outcome in 32 enrolled ALL patients is shown in Figure 1.

\section{Discussion}

Despite the challenging toxicity profile, incorporation of PEG-ASP in pediatric and adult ALL protocols remains a worldwide therapeutic standard $[1,3,4]$ Therefore, our ALL regimen included a single administration of PEG-ASP at a dose of $1000 \mathrm{IU} / \mathrm{m}^{2}$ (median of $1800 \mathrm{IU}$ ) and involved only patients with undetectable Philadelphia chromosome. We observed 4 induction deaths (13\%) and these were likely related to PEG-ASP use. All deaths were in patients $>50$ years at diagnosis and occurred at a median 5 days after PEG-ASP. This induction death rate was similar to that determined in other study groups [4]. In contrast to the UKALL14 study [4] where bacterial sepsis was the main cause of death, our expired patients had negative bacterial and fungal cultures. Moreover, CRP was only slightly elevated at the time of death. However, the concomitant presence of grade 3/4 PEG-ASPrelated hepatotoxicity was demonstrated in 3 of the 4 patients who died. Similar findings were also demonstrated in the UKALL14 study; PEG-ASP associated toxicity was present in 8 of 16 induction deaths and grade $3 / 4$ hyperbilirubinemia was the most common adverse effect.
In a more recent analysis, 122 newly diagnosed standardrisk ALL patients received E. coli-asparaginase or PEG-ASP induction according to MRC UKALL12 E2993 protocol. There was no difference in demographic and laboratory parameters at study entry, and OS rates were also comparable. 3 deaths occurred in the PEG-ASP cohort compared to no fatal events in the E. coli-asparaginase group. However, no treatment-related mortality events were considered related to asparaginase use.

The most common grade 3/4 PEG-ASP-related toxicities were hyperfibrinogenemia (23\%), hypoalbuminemia (21\%) and hypertriglyceridemia (15\%). Grade 3 and 4 hyperbilirubinemia and transaminitis were demonstrated in 13\% of patients. Severe pancreatitis and deep vein thrombosis (DVT) occurred in $6 \%$, and $2 \%$ treated subjects, respectively. However, it is important that the incidence and severity of adverse events did not differ between PEG-ASP and E. coli-asparaginase treatments [6]. Hepatotoxicity was

Table 2. Laboratory adverse reactions reported with PEG-ASP therapy in Study Product Characteristics.

\begin{tabular}{l|c|c}
\hline Event term/No of tested patients & $\begin{array}{c}\text { AE grade 1-2 } \\
\text { (No/\%) }\end{array}$ & $\begin{array}{c}\text { AE grade 3-4 } \\
\text { (No/\%) }\end{array}$ \\
\hline Liver function & & \\
Bilirubin increased (29) & $15(52)$ & $9(31)$ \\
ALT increased (30) & $15(50)$ & $3(10)$ \\
AST increased (29) & $6(21)$ & $2(7)$ \\
FA increased (22) & $5(23)$ & $3(14)$ \\
GGTP increased (26) & $8(31)$ & $7(27)$ \\
Pancreas function & & \\
Amylase increased (15) & $1(7)$ & $1(7)$ \\
Coagulation tests & & \\
APTT prolongation (32) & $18(56)$ & $1(3)$ \\
PT prolongation (26) & $3(12)$ & 0 \\
Fibrinogen decreased (29) & $8(28)$ & $17(58)$ \\
\hline
\end{tabular}

Legend: $\mathrm{ALT}=$ alanine aminotransferase; APTT=activated partial thrombin time; $\mathrm{ASP}=$ aspartate aminotransferase; GGTP= gamma glutamyl transpeptidase; $\mathrm{FA}=$ alkaline phosphatase; $\mathrm{PT}=$ prothrombin time

Table 3. Summary of deaths during induction.

\begin{tabular}{|c|c|c|c|c|c|c|c|}
\hline $\begin{array}{l}\text { Patient } \\
\text { initials }\end{array}$ & $\begin{array}{c}\text { Age } \\
\text { (years) }\end{array}$ & Sex & Co-morbidities & $\begin{array}{l}\text { Total PEG-ASP } \\
\text { dose (IU) }\end{array}$ & $\begin{array}{c}\text { Days from } \\
\text { PEG-ASP dose }\end{array}$ & $\begin{array}{c}\text { AE grade } 3-4 \\
\text { post PEG-ASP }\end{array}$ & Clinical manifestations \\
\hline SD & 58 & M & $\begin{array}{l}\text { hypertension; hyperuricemia } \\
(\mathrm{BMI}=24)\end{array}$ & 1650 & 6 & $\begin{array}{l}\text { bilirubin, GGTP, } \\
\text { fibrinogen }\end{array}$ & Intracranial hemorrhage \\
\hline MB & 59 & $\mathrm{~F}$ & $\begin{array}{l}\text { hypertension, diabetes, obe- } \\
\text { sity }(\mathrm{BMI}=40)\end{array}$ & 2000 & 1 & none & $\begin{array}{l}\text { Abdominal pain, diarrhea, } \\
\text { shock }\end{array}$ \\
\hline IS & 52 & $\mathrm{~F}$ & $\begin{array}{l}\text { hypertension, diabetes, } \\
\text { obesity (BMI=53), history of } \\
\text { uterus cancer }\end{array}$ & 2200 & 9 & $\begin{array}{l}\text { bilirubin, } \\
\text { fibrinogen }\end{array}$ & $\begin{array}{l}\text { Abdominal pain, hepatic-renal } \\
\text { syndrome, shock }\end{array}$ \\
\hline $\mathrm{ZK}$ & 61 & M & $\begin{array}{l}\text { hypertension, obesity } \\
\text { (BMI=47), psoriasis }\end{array}$ & 2000 & 4 & Bilirubin, GGTP & Abdominal pain, fever, shock \\
\hline
\end{tabular}

Legend: $\mathrm{BMI}=$ body mass index; $\mathrm{GGTP}=$ gamma glutamyl transpeptidase, $\mathrm{M}=$ male; $\mathrm{F}=$ female 


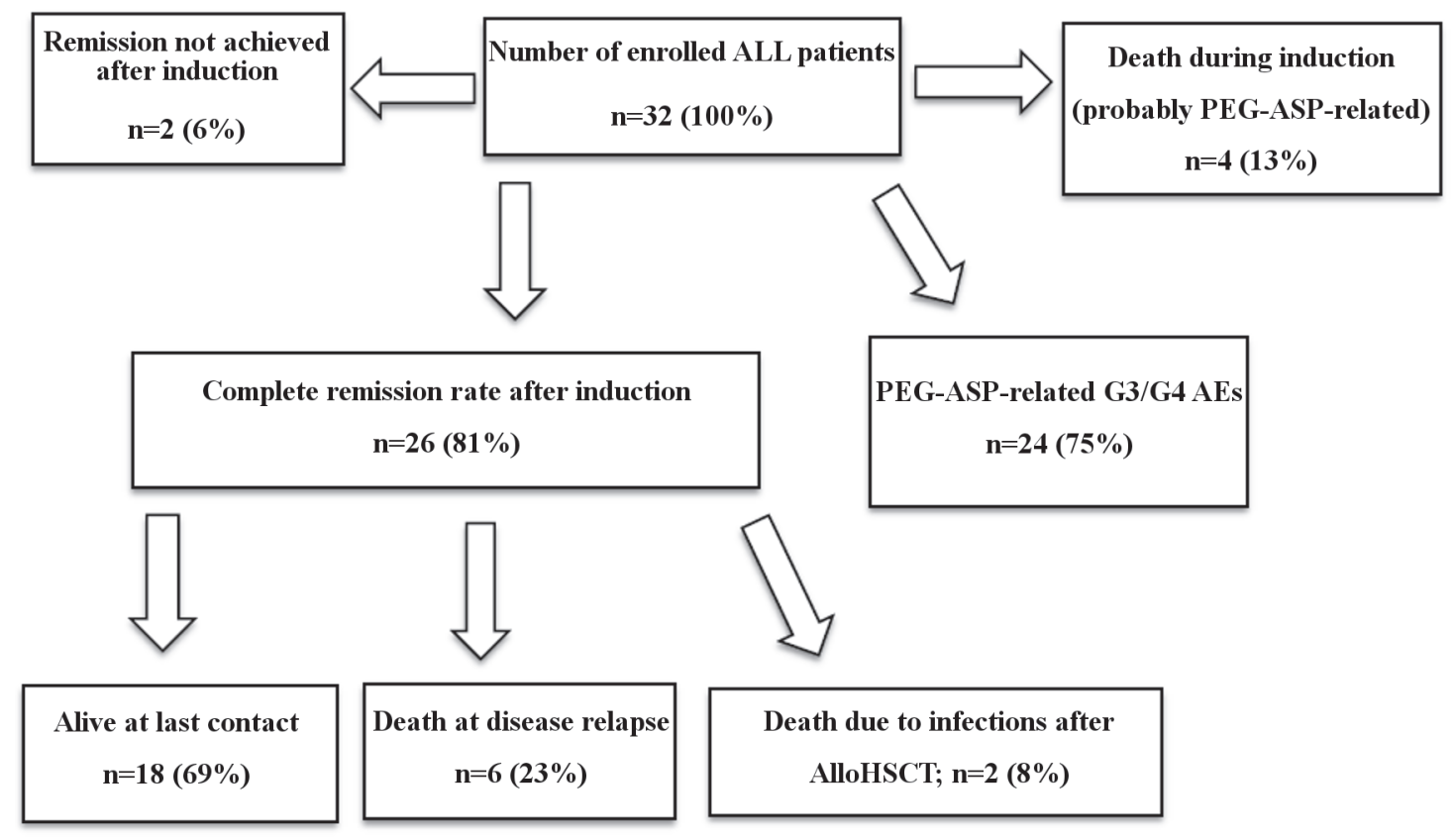

Figure 1. Flow chart of 32 enrolled patients with acute lymphoblastic leukemia. AEs=adverse events; ALL=acute lymphoblastic leukemia; AlloHSCT=allogeneic stem cell transplantation; PEG-ASP: pegylated asparaginase

also the most frequent PEG-ASP-related toxicity in a recent UKALL14 study [4]. In addition, 36\% treated patients developed grade $3 / 4$ liver dysfunction and elevated levels of serum bilirubin (23\%) and alkaline phosphatase (20\%) were the most common. Age $>40$ years and BMI were the only factors associated with the occurrence of grade $3 / 4$ hepatotoxicity.

Hepatotoxicity was also the most common finding in our study and pancreatitis and DVT were rarely observed. Grade $3 / 4$ hyperbilirubinemia (31\%), increased gamma glutamyl transpeptidase (27\%) and decreased fibrinogen (58\%) were the most frequent abnormalities, and pancreas dysfunction and thrombotic episodes were only sporadic.

Several risk factors which may predict the occurrence of PEG-ASP-related hepatotoxicity in ALL patients have recently been identified. These include: body surface area $>2 \mathrm{~m}^{2}$, albumin concentration $<3 \mathrm{mg} / \mathrm{dl}$ and platelet count $<50 \times 10^{9} / 1$. It is noteworthy that the 30 -day mortality was significantly higher for patients who developed hepatotoxicity than in those without liver damage [7]. In contrast to above-mentioned studies, only single patients developed grade 3/4 PEG-ASP-related toxicities in an analysis reported by Douer et al.; where hyperbilirubinemia and transaminitis were found in $8 \%$ and $12 \%$ of patients, respectively. Although decreased fibrinogen and antithrombin concentrations were not associated with bleeding events, cryoprecipitate was prophylactically administered [6]. This supports our findings that no bleeding events were observed in our patient population despite severe hyperfibrinogenemia and decreased AT level after PEG-ASP in some tested patients.
The occurrence of liver toxicity during remission induction determines subsequent on-time delivery of chemotherapy. In fact, chemotherapy was discontinued or delayed in 7 patients in our study and this agrees with other reports $[4,6]$. However, it should be noted that most PEG-ASP toxic effects are manageable and reversible.

While the patomechanism of hepatotoxicity of asparaginase is not fully understood, it has been suggested that asparaginase itself is not hepatotoxic, and that this result could be partly due to contamination by bacterial endotoxin. Other implicated factors include co-infection, co-morbidities and concomitant use of other hepatotoxic agents [8].

In the treatment of ALL patients, one must be aware of possible overlapping toxicity of PEG-ASP and other agents administered in remission induction. In our study, the induction deaths happened early (median of 5 days) after single administration of PEG-ASP dose (D10 or D13 depending on age group). This toxicity overlaps with the anthracycline dose given twice before and twice after PEG-ASP (D1, D8, D15 and D22). This finding agrees with the UKALL14 study where most deaths were early, and observed after a single PEG-ASP dose on Day 4. However, most of those fatal events were caused by septic shock [4], and in contrast, although our patient cohort exhibited grade 4 neutropenia and thrombocytopenia, none of the patients who died had infection symptoms at the time of their death.

In the light of the current knowledge, it is difficult to draw definite conclusions about PEG-ASP toxicity overlap with other agents used in ALL induction. However, this appears to 
be partly due to different PEG-ASP and anthracycline dose and schedules throughout the studies and the variations in patient characteristics at study entry. Moreover, some reports included ALL with detectable Philadelphia chromosome, and the patients still received imatinib which is widely known to be hepatotoxic $[4,5,9]$.

Studies also confirm that the risk of PEG-ASP-related toxicity is greatest during the first treatment cycle; most likely due to a high level of tumor-related circulating cytokines and the concomitant use of steroids [10].

The UKALL14 study identified $>40$ years of age as the predictor of induction death and liver-toxicity [4], and this is supported in other studies [11]. Further, omitting PEG-ASP and reducing the daunorubicin dose in the ALL treatment protocol for older patients resulted in decreased induction death [4].

Aldoss et al. report that BMI is another significant factor associated with hepatotoxicity in the UKALL14 study [4]. Elevated BMI and obesity were strongly associated with the occurrence of grade $3 / 4$ hyperbilirubinemia, transaminitis and hypertriglyceridemia, but not with hyperfibrinogenemia [10]. These results support our findings where the four patients who died were over 50 years of age, and 3 of these had massive obesity.

The association between obesity and hepatotoxicity requires clarification. It was found in animal models that the presence of hepatic steatosis predisposes to PEG-ASP-related hepatotoxicity [12].

In conclusion, herein we demonstrated that PEG-ASP administered in induction remission for ALL patients resulted in significant, but mostly reversible, liver-toxicity and this caused a delay in subsequent chemotherapy. While the combination of PEG-ASP with other myelo-suppressive agents may have been responsible for the overlapping toxicity, PEG-ASP treatment should be administered with caution in older, obese patients with acute lymphoblastic leukemia.

Acknowledgements: We appreciate the Polish Adult Leukemia Group for initiation and delivery of the ALL6 treatment protocol (group leader: Prof. Sebastian Giebel).

\section{References}

[1] GRAHAM ML. Pegaspargase: a review of clinical studies. Adv Drug Deliv Rev 2003; 55: 1293-1302. https://doi. org/10.1016/S0169-409X(03)00110-8

[2] AVRAMIS VI, SPENCE SA. Clinical pharmacology of asparaginases in the United States: asparaginase population pharmacokinetic and pharmacodynamics (PK-PD) models
(NONMEM) in adult and pediatric ALL patients. J Pediatr Hematol Oncol 2007; 29: 239-247. https://doi.org/10.1097/ MPH.0b013e318047b79d

[3] ETTINGER LJ, KURTZBERG J, VOUTE PA, JURGENS H, HALPERN SL. An open-label, multicenter study of polyethylene glycol-asparaginase for the treatment of acute lymphoblastic leukemia. Cancer 1995; 75: 1176-1181.

[4] PATEL B, KIRKWOOD AA, DEY A, MARKS DI, MCMILLAN AK et al. Pegylated-asparaginase during induction therapy for adult acute lymphoblastic leukemia: toxicity data from the UKALL14 trial. Leukemia 2017; 31: 58-64. https:// doi.org/10.1038/leu.2016.219

[5] DOUER D, YAMPOLSKY H, COHEN LJ, WATKINS K, LEVINE AM et al. Pharmacodynamics and safety of intravenous pegaspargase during remission induction in adults aged 55 years or younger with newly diagnosed acute lymphoblastic leukemia. Blood 2007; 109: 2744-2750. https:// doi.org/10.1182/blood-2006-07-035006

[6] LIU WJ, WANG H, WANG WD, ZHU MY, LIU CC et al. Use of PEG-asparaginase in newly diagnosed adults with standrad-risk acute lymphoblastic leukemia compared with E. coli- asparaginase: a retrospective single-center study. Sci. Rep 2016; 6: 39463. https://doi.org/10.1038/srep39463

[7] RAUSCH CR, MARINI BL, BENITEZ LL, ELIAS A, BURKE $\mathrm{PW}$ et al. PEGging down risk factors for peg-asparaginase hepatotoxicity in patients with acute lymphoblastic leukemia. Leuk Lymphoma 2018; 59: 617-624. https://doi.org/10. 1080/10428194.2017.1349902

[8] SCHEIN PS, RAKIETEN N, GORDON BM, DAVIS RD, RALL DP. The toxicity of Escherichia-coli L-asparaginase. Cancer Res 1969; 29: 426-434.

[9] GOEKBUGET N, BAUMANN A, BECK J, BRUEGGEMANN M, DIEDRICH $\mathrm{H}$ et al. PEG-Asparaginase intensification in adult acute lymphoblastic leukemia (ALL): significant improvement of outcome with moderate increase of liver toxicity in the German Multicenter Study Group for Adult ALL (GMALL) Study 07/2003. Blood 2010; 116: 494.

[10] ALDOSS I, DOUER D, BEHRENDT CE, CHAUDHARY P, MOHRBACHER A et al. Toxicity profile of repeated doses of PEG-asparaginase incorporated into a pediatric-type regimen for adult acute lymphoblastic leukemia. Eur J Haematol 2015; 96: 375-380. https://doi.org/10.1111/ejh.12600

[11] HUGUET F, LEGUAY T, RAFFOUX E, THOMAS X, BEDJORD K et al. Pediatric-inspired therapy in adults with Philadelphia chromosome-negative acute lymphoblastic leukemia: the GRALL-2003 study. J Clin Oncol 2009; 27: 911-918. https://doi.org/10.1200/JCO.2008.18.6916

[12] ROESMANN A, ATIFY M, PANSE J, EISERT A, STEITZ J et al. L-carnitine ameliorates L-asparaginase-induced acute liver toxicity in steatotic rat livers. Chemotherapy 2013; 59: 167-175. https://doi.org/10.1159/000353402 